\title{
Vaginopathic and proteolytic Candida species in outpatients attending a gynaecology clinic
}

\author{
L Agatensi, F Franchi, F Mondello, R L Bevilacqua, T Ceddia, F De Bernardis, \\ A Cassone
}

\begin{abstract}
Non-pregnant, non-diabetic outpatients were examined for the presence of pathogenic vaginal yeasts to determine if a correlation existed between a specific yeast and clinical disease. Yeasts were isolated as single vaginal species from 186 of 228 subjects with clinically diagnosed candidal vaginitis, as well as from 122 out of $\mathbf{3 8 0}$ asymptomatic, agematched controls. Apart from Candida albicans and $\boldsymbol{C}$ glabrata, other prevalent species were $C$ krusei, $C$ parapsilosis and Saccharomyces cerevisiae which accounted for $9 \cdot 2 \%, 6 \cdot 0 \%$ and $5 \cdot 4 \%$, and $9.0 \%, 2.4 \%$ and $19.7 \%$, of yeasts from patients and carriers, respectively. Only $C$ albicans and $C$ parapsilosis were significantly more common in those with vaginitis. Only the isolates of these two species secreted aspartyl proteinase in vitro, and the amount of the enzymes secreted by the isolates from patients was significantly higher than that secreted by the isolates from carriers. These two species consistently produced vaginal infection in pseudoestrus rats, whereas none of the non-proteolytic species tested ( $C$ glabrata, $C$ krusei, and $S$ cerevisiae) colonised the vagina in these rats.
\end{abstract}

Proteinase secretion correlated with experimental vaginal infection; it could also be a reliable factor for distinguishing clinically active infection from asymptomatic fungal carriage.

Candidal vaginitis continues to pose several aetiological, diagnostic, and pathogenetic challenges. ${ }^{1-3}$ While many authors have estimated the incidence of candidal vaginitis and recorded the species of Candida more often among patients with vaginitis, reports based on careful clinical and mycological observations are relatively few. $C$ albicans and $C$ glabrata are believed to be the only or the most common agents of candidal vaginitis, although many other species of this fungus have been isolated from the vagina of infected subjects. ${ }^{1}$ In particular, $C$ parapsilosis has recently been shown to have considerable pathogenic potential in experimentally induced infection in pseudoestrus rats whose ovaries had been removed. ${ }^{45}$

No agreement has been reached as to whether the presence of even the most pathogenic species ( $C$ albicans) in the vagina is always associated with vaginitis, or whether carriage of Candida sp is normal but harmless. For some authors $C$ albicans is rarely found in the vagina in the absence of local pathology. ${ }^{1}$ McCormack et al observed symptoms in $67 \%$ of women whose vaginal cultures contained yeasts and they concluded that vaginal colonisation by yeasts is commonly associated with vulvovaginal symptoms. ${ }^{6}$ On the other hand, other authors have convincingly shown that the prevalence of vaginal yeasts was similar in women with and without vaginal symptoms. ${ }^{78}$ Odds $^{1}$ and Sobel ${ }^{39}$ have recently reviewed the evidence for normal vaginal carriage of Candida sp.

The pathogenesis of candidal vaginitis is also unclear. Although several risk factors have been identified, such as pregnancy and diabetes, and treatment with corticosteroids and broad spectrum antibiotics, ${ }^{19}$ little is known about the virulence factors of the yeast responsible for vaginal colonisation or vaginitis, and the mechanisms which determine the transition from asymptomatic yeast carriage to vaginitis.

\section{Methods}

Non-pregnant, non-diabetic women $(\mathrm{n}=608)$, aged 19 to 63 years and attending the Center for Tumor Prevention in Sant'Andrea Hospital, Rome, were enrolled in this study. The criteria for inclusion were as follows. The first five women who presented to the Center each day between 1986 and 89, asking for a gynaecological examination for any reason, except those who were: (i) younger than 18 and 65 years or older; (ii) pregnant; (iii) diabetic; (iv) taking antibiotics, or topical antifungal agents two months before examination; (v) taking vaginal contraceptives the week preceding the visit to the Center. Each subject completed a questionnaire on demographic, social, sexual and medical data. Around 10\% of women regularly used contraception, a percentage that was similar among patients and controls.

All subjects with non-candidal vaginitis (based on the criteria reported by Sweet ${ }^{10}$ ), as well as those from whose vagina more than a single yeast species was isolated, were excluded. The differential diagnosis of candidal vaginitis was based on the isolation of a yeast species from subjects with a typical vaginal discharge (clumpy, cottage-cheese appearance) and intense vulvovaginal pruritis, accompanied by vulvar erythema and dyspareunia. All other yeast positive (by culture) asymptomatic subjects served as controls. All eligible subjects gave their written, informed consent.
Bacteriology and Medical

di Sanità, Viale Regina

Italy

Accepted for publication 22 May 1991 
YEAST ISOLATION AND IDENTIFICATION

Two high vaginal samples were taken with cotton tipped swabs from all patients. One swab was immediately smeared on to a glass slide, fixed, and stained with periodic acidSchiff (PAS) for microscopical examination. The other was kept in sterile physiological saline and transferred to the laboratory where it was seeded on plates of Sabouraud-dextrose agar (BBL, Baltimore, Maryland) with added chloramphenicol (50 $\mu \mathrm{g} / \mathrm{ml})$ and Mycosel agar and then immersed into enrichment broth. All cultures were incubated for 48-72 hours at $30^{\circ} \mathrm{C}$. All isolates were preliminarily identified on the basis of their assimilation or fermentation profiles in the API 20C system (BioMèrieux), capacity to form germ-tubes in serum, morphology and chlamydospores in corn-meal agar. The identification was confirmed by an expanded battery of assimilationfermentation tests according to Meyer et al. ${ }^{11}$ Serological analysis according to Tsuchiya $e t$ $a l^{12}$ was also performed as an aid to the final identification and serotyping of $C$ albicans.

\section{PROTEINASE SECRETION IN VITRO}

All isolates were tested for their ability to grow and produce clear zones of hydrolysis in bovine serum albumin (BSA) agar using previously established methods. ${ }^{13}$ Acid proteinase activity was scored as follows: - or \pm when no visible or a very limited clarification of the agar around the colony was present; $1+$ when visible proteolysis was observed (1-2 mm); and $2+$ when agar clarification largely exceeded (3-5 $\mathrm{mm}$ ) the margin of the colony. Several isolates of $C$ albicans and all isolates of $C$ parapsilosis were also tested for proteinase assay in liquid medium containing bovine haemoglobin, as described previously. ${ }^{1314}$

\section{EXPERIMENTAL VAGINITIS IN RATS}

For experimentally induced vaginal infections, female Wistar rats, weighing $80-100 \mathrm{~g}$, whose ovaries had been removed, were obtained from Charles River Breeding Laboratories and injected subcutaneously with $0.5 \mathrm{mg}$ of oestradiol benzoate (Benzatrone; Samil, Rome, Italy) every two days. Six days after the first dose of oestradiol the animals were inoculated intravaginally with $10^{7}$ cells $(0 \cdot 1 \mathrm{ml}$ volume) of each yeast isolate tested. The yeast had been grown to stationary phase in YEPD medium at $28^{\circ} \mathrm{C} .^{5}$ It was then harvested by low speed centrifugation, washed, and suspended to the required number in phosphate buffered saline. Yeast cells were injected into the vaginal cavity through a syringe equipped with a multipurpose calibrated tip (Combitip; PBI, Milan, Italy). Vaginal fluid was taken from each animal every two days, with a special purpose calibrated $(1 \mu \mathrm{l})$ plastic loop (Dispoinoc; PBI). Some aliquots were used for microscopical examination; other specimens (one vaginal sample per rat per culture) were used for quantitation of vaginal colonisation. For this, the contents of each loop were vigorously shaken in $0.1 \mathrm{ml}$ of phosphate buffered saline and the portions streaked over plates containing Sabouraud glucose agar plus chloram- phenicol and incubated for 48-72 hours at $28^{\circ} \mathrm{C}$. At intervals during vaginal infection, colonies developed on the mycological medium were subjected to confirmatory species identification according to the diagnostic criteria reported above.

Differences in the frequency with which each yeast species was isolated from the different groups were assessed for significance using the $\chi^{2}$ method. Differences in proteinase secretion in liquid medium were assessed by Student's $t$ test; whereas the differences in proteinase secretion in BSA agar were assessed by the $\chi^{2}$ test or Fisher's exact probability methods, as appropriate. Differences in yeast cell counts (CFU) in infected vaginal fluids were assessed by Student's $t$ test.

\section{Results}

PREVALENCE AND DISTRIBUTION OF VAGINAL YEAST SPECIES

Yeasts were isolated as single vaginal species from 186 of 228 patients with clinical symptoms and signs of candidal vaginitis. Either two or more yeasts (generally $C$ albicans plus $C$ krusei, or $C$ parapsilosis; $\mathrm{n}=10$ subjects) or no yeast ( $n=32$ subjects) were isolated from the remaining 42 patients. Yeasts were also isolated as single vaginal species from 122 of 380 asymptomatic subjects, the remaining 258 being negative for vaginal yeast isolation. Table 1 shows the distribution of species and their relative proportions in the two groups of subjects.

In the group with vaginitis $C$ albicans was the predominant species with a prevalence of almost $50 \%$. As expected, the second most frequently isolated species was $C$ glabrata (slightly more than $20 \%$ ), followed by, in descending rank of prevalence, $C$ krusei, $C$ parapsilosis, $C$ kefyr and $C$ tropicalis. Saccharomyces cerevisiae and a miscellanea of other non-pathogenic Candida species represented only $7.5 \%$ of all isolates. Among asymptomatic, control subjects $C$ albicans and $C$ glabrata were isolated with a similar frequency (around 20\%), which was also similar to the percentage isolation of $S$ cerevisiae or miscellaneous yeast species. Overall, the seven aforementioned yeast species accounted for more than $92 \%$ of all isolations.

Table 1 also shows that $C$ albicans and $C$ parapsilosis were isolated significantly more frequently from those with vaginitis than from carriers; the reverse was true for $S$ cerevisiae and the miscellaneous yeasts: in particular, Rhodotorula spp were isolated only from asymptomatic subjects. There was no significant difference between the two groups of subjects examined in terms of the isolation prevalence of all other species, including $C$ glabrata (table 1).

\section{IN VITRO PROTEINASE SECRETION}

The ability to secrete an acid (aspartyl) proteinase, a potential virulence factor in the pathogenesis of candidal vaginitis, ${ }^{1314}$ was assessed in vitro using both BSA agar (BSA-P) and haemoglobin bovine liquid medium (HB-P). ${ }^{14}$ 
Table 1 Correlation between clinical vaginitis, vaginal carriage, and yeast isolation

\begin{tabular}{|c|c|c|c|c|c|}
\hline \multirow[b]{2}{*}{ Yeasts isolated } & \multicolumn{2}{|c|}{$\begin{array}{l}\text { Women with signs and } \\
\text { symptoms of candidal } \\
\text { vaginitis }\end{array}$} & \multicolumn{2}{|c|}{$\begin{array}{l}\text { Asymptomatic women } \\
\text { (carriers) }\end{array}$} & \multirow[b]{2}{*}{ p value } \\
\hline & $N=$ & $\%$ Isolates & $N=$ & $\%$ Isolates & \\
\hline $\begin{array}{l}\text { Candida albicans } \\
\text { Candida glabrata } \\
\text { Candida krusei } \\
\text { Candida parapsilosis } \\
\text { Candida kefyr } \\
\text { Candida tropicalis } \\
\text { Saccharomyces cerevisiae } \\
\text { Others }\end{array}$ & $\begin{array}{c}92 \\
41 \\
17 \\
13 \\
5 \\
4 \\
10 \\
4^{\star}\end{array}$ & $\begin{array}{r}49 \cdot 9 \\
22 \cdot 3 \\
9 \cdot 2 \\
6 \cdot 0 \\
2 \cdot 7 \\
2 \cdot 1 \\
5 \cdot 4 \\
2 \cdot 1\end{array}$ & $\begin{array}{r}24 \\
26 \\
11 \\
3 \\
7 \\
5 \\
24 \\
22 \dagger\end{array}$ & $\begin{array}{r}19 \cdot 7 \\
21 \cdot 3 \\
9 \cdot 0 \\
2 \cdot 4 \\
5 \cdot 7 \\
4 \cdot 1 \\
19 \cdot 7 \\
18 \cdot 0\end{array}$ & $\begin{array}{l}<0.01 \\
\text { NS } \\
\text { NS } \\
<0.05 \\
\text { NS } \\
\text { NS } \\
<0.05 \\
<0.01\end{array}$ \\
\hline Total & 186 & & 122 & & \\
\hline
\end{tabular}

${ }^{\star} C$ famata 1,3 unidentified. $\dagger$ Rhodotorula sp. 13,C famata 2, C humicola 1, C lipolytica 1, C lambica 1, Trichosporon beigelii
1, Aureobasidium sp 1.

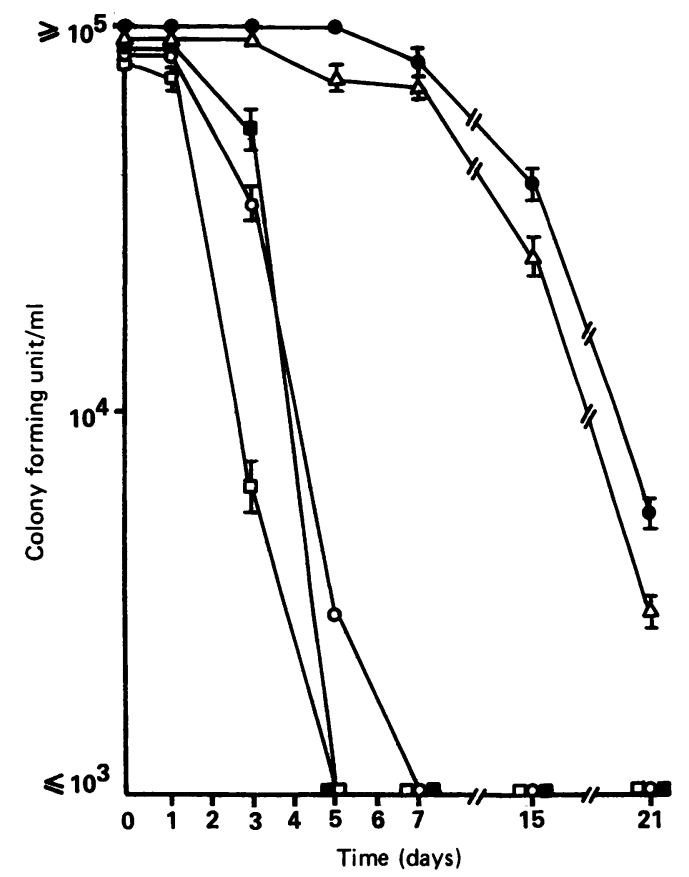

Experimentally induced rat vaginitis with vaginal isolates of various Candida species. Groups of five rats were infected (day 0 ) with $10^{7}$ cells of Candida albicans (isolate $S A-40 ;-0), C$ parapsilosis ( $S A-19 ; \triangle-$ $\triangle$ ), Cglabrata ( $S A-380$; - $), C$ krusei ( $S A-301$; $O-O)$, and $S$ cerevisiae ( $S A-264 ; \square-\square$ ).

Candida numbers are expressed as mean CFU (SE) (bar) $/ \mathrm{ml}$ of vaginal fuid of infected $\left(\geq 10^{3} \mathrm{CFU} / \mathrm{ml}\right)$ rats. those from carriers; no significant difference was noticed between the proteinase activity of $C$ albicans and that of $C$ parapsilosis, regardless of their isolation source.

EXPERIMENTALLY INDUCED RAT VAGINITIS

While the potential of both $C$ albicans and $C$ parapsilosis to produce vaginal infection in rats without ovaries and maintained with oestradiol exist on the experimental potential to cause vaginitis of $C$ glabrata, $C$ krusei, or $S$ cerevisiae. Because of the high prevalence of these three is already established, ${ }^{514}$ no or very few data

Table 2 Acid proteinase activity ${ }^{13}$ in vitro by vaginal isolates of Candida spp

\begin{tabular}{|c|c|c|c|c|c|c|c|c|c|c|}
\hline \multirow[b]{3}{*}{ Species } & \multirow{3}{*}{$\begin{array}{l}\text { No of isolates } \\
\text { tested }\end{array}$} & \multicolumn{6}{|c|}{$B S A-P$ of isolates from: } & \multicolumn{2}{|c|}{$H B-P(\Delta O D / m l)$ of isolates from: } & \multirow{3}{*}{$t$ test } \\
\hline & & \multicolumn{3}{|c|}{ Patients } & \multicolumn{3}{|c|}{ Controls } & \multirow{2}{*}{$\begin{array}{l}\text { Patients } \\
\text { (mean }(S D))\end{array}$} & \multirow{2}{*}{$\begin{array}{l}\text { Controls } \\
\text { (mean (SD).) }\end{array}$} & \\
\hline & & $2+$ & $1+$ & 0 & $2+$ & $1+$ & 0 & & & \\
\hline $\begin{array}{l}\text { Calbicans } \\
\text { C parapsilosis } \\
\text { Cglabrata } \\
\text { C krusei } \\
\text { C tropicalis } \\
C \text { kefyr }\end{array}$ & $\begin{array}{r}112 \\
17 \\
78 \\
34 \\
9 \\
11\end{array}$ & $\begin{array}{r}44 \\
9 \\
0 \\
0 \\
0 \\
0\end{array}$ & $\begin{array}{l}34 \star \star \\
1 \neq \ddagger \\
0 \\
0 \\
3 \\
2\end{array}$ & $\begin{array}{r}0 \\
1 \\
41 \\
17 \\
1 \\
3\end{array}$ & $\begin{array}{l}3 \\
1 \\
0 \\
0 \\
0 \\
0\end{array}$ & $\begin{array}{l}31 \star \star \\
5 \neq \ddagger \\
0 \\
0 \\
1 \\
1\end{array}$ & $\begin{array}{r}0 \\
0 \\
37 \\
17 \\
4 \\
5\end{array}$ & $\begin{array}{l}1.06(0.24) \star \\
1.14(0.33) \ddagger\end{array}$ & $\begin{array}{l}0.78(0.12) \dagger \\
0.80(0.13) \dagger \dagger\end{array}$ & $\begin{array}{l}\mathrm{p}<0.001 \\
\mathrm{p}<0.01\end{array}$ \\
\hline
\end{tabular}

$\star 50$ different isolates, 70 independent determinations.

+34 isolates, 38 independent determinations.

$\ddagger 11$ isolates, 12 determinations.

$\dagger+3$ isolates, 8 determinations.

$\star \star \mathrm{p}<0.01, \chi^{2}$ test, comparing the ratios $2+: 1+$.

$\ddagger \mathrm{p}<0.05$, Fisher's exact test, comparing the ratios $2+: 1+$

Table 3 Quantitation of vaginal colonisation (CFU (SD) $\times 10^{-3}$ yeast cells/ml vaginal fluid) in pseudoestrus rats infected with Candida isolates

\begin{tabular}{|c|c|c|c|c|c|}
\hline \multirow[b]{2}{*}{ Fungal isolate } & \multicolumn{5}{|l|}{ Days after challenge } \\
\hline & 1 & 2 & 7 & 14 & 21 \\
\hline $\begin{array}{l}{ }^{\star} \text { Calbicans } \\
{ }^{\star} C \text { parapsilosis } \\
C \text { glabrata } \\
C \text { glabrata } \\
{ }^{\star} \text { Cglabrata } \\
C \text { glabrata } \\
{ }^{\star} \text { C krusei } \\
C \text { krusei } \\
C \text { krusei } \\
\star S \text { cerevisiae } \\
C \text { krusei }\end{array}$ & $\begin{array}{l}>100(5 / 5) \dagger \\
>100(5 / 5) \\
>100(5 / 5) \\
57 \cdot 2(17 \cdot 9)(5 / 5) \\
>100(5 / 5) \\
>100(5 / 5) \\
>100(5 / 5) \\
29(17 \cdot 2)(4 / 5) \\
>100(5 / 5) \\
76(20 \cdot 8)(4 / 5) \\
58(13 \cdot 2)(5 / 5)\end{array}$ & $\begin{array}{c}>100(5 / 5) \\
>100(5 / 5) \\
15 \cdot 8(4 \cdot 0)(5 / 5) \\
16 \cdot 0(1 \cdot 8)(4 / 5) \\
55 \cdot 4(19)(5 / 5) \\
20 \cdot 0(4 \cdot 0)(4 / 5) \\
34(16 \cdot 0)(4 / 5) \\
24 \cdot 2(22)(3 / 5) \\
10 \cdot 6(5 \cdot 6)(5 / 5)\end{array}$ & $\begin{aligned} & 80(2 \cdot 5)(5 / 5) \\
< & 1(0 / 5) \\
< & 1(0 / 5) \\
< & 1(0 / 5) \\
& 1(1 / 5) \\
< & 1(0 / 5) \\
< & 1(0 / 5) \\
< & 1(0 / 5) \\
& 2(1 / 5)\end{aligned}$ & $\begin{array}{l}\quad 40(3 \cdot 8)(5 / 5) \\
23 \cdot 7(2 \cdot 1)(2 / 5) \\
<1(0 / 5) \\
<1(0.5)\end{array}$ & $\begin{array}{l}26(2.5)(3 / 5) \\
19(3.7)(2 / 5) \\
<1(0 / 5) \\
<1(0 / 5)\end{array}$ \\
\hline
\end{tabular}

* Shown in kinetics, in an independent experiment, in the figure.

†Rats infected/total. 
yeasts in our subjects, we tested randomly selected isolates of these three species in a previously established rat vaginitis model, and compared them with two isolates of $C$ albicans and $C$ parapsilosis as positive controls.

Table 3 summarises the individual data obtained for each isolate, inclusive of both the total cfu counts and the number of rats infected ( $>10^{3}$ cells $/ \mathrm{ml}$ of vaginal fluid) over total numbers, over three weeks, after vaginal challenge of $10^{7}$ cells. The figure outlines the kinetics of infection with representative isolates of each species. Altogether, the data show clearly that the isolates of $C$ krusei and $C$ glabrata, like those of $S$ cerevisiae, were eliminated from the vagina of infected rats within the first week after intravaginal challenge. On the contrary, both $C$ parapsilosis and $C$ albicans persisted to infective concentrations $\left(>10^{3}\right.$ $\mathrm{cfu} / \mathrm{ml}$ ), significantly longer, confirming previous results. No significant differences were found among the various isolates of $C$ glabrata or $C$ krusei in the rate of elimination of yeast cells from the vagina, measured both as total cfu count and as a proportion of infected rats.

\section{Discussion}

One hundred and eighty six cases of clinically and mycologically defined candidal vaginitis were matched with 122 asymptomatic carriers among subjects attending a gynaecological Center in Rome. All the women were not pregnant and non-diabetic, and no difference between the two groups of subjects was present in the use of method of birth control. Thus the results may offer a reasonable indication of the prevalence of defined yeast species in vaginitis, as opposed to vaginal carriage of the same species, in the population examined. Despite a large number of studies, ${ }^{1}$ very few attempts have been made to evaluate prevalence and role of vaginal isolates other than $C$ albicans as pathogenic agents, and to assess their virulence and experimental pathogenicity in animals.

A remarkable aspect of the population we examined was that about half the yeasts isolated from typical cases of candidal vaginitis were not $C$ albicans, and some were non-Candida (Saccharomyces) species. The increased proportion of these apparently uncommon, vaginal species allowed us to evaluate preferential association of these species with carriage or overt disease. Interestingly, a well known aetiological agent of candidal vaginitis, $C$ glabrata, had a high comparable prevalence in both patients and carriers, whereas only $C$ parapsilosis resembled $C$ albicans in being significantly more frequently isolated from patients than carriers. This strongly suggests a correlation between $C$ parapsilosis and candidal vaginitis, in keeping with our previous results obtained in oophorectomised, pseudoestrus rats. ${ }^{45}$ This also suggests that both $C$ albicans and $C$ parapsilosis may have special virulence characteristics which facilitate the transition from harmless carriage to pathogenicity. One possible virulence factor which seems to belong to these two species and to differentiate them from all other prevalent vaginal species is the secretion of an aspartyl proteinase, an enzyme capable of hydrolysing mucosal IgA, the most effective barrier against infection in the vagina. ${ }^{15}$ In this survey we extended previous observations ${ }^{513}$ to show that all vaginal isolates of $C$ albicans and $C$ parapsilosis produce a remarkable amount of proteinase in vitro. Importantly, acid proteinase secretion by the isolates from the patients with vaginitis was significantly higher than that by the isolates from controls, confirming preliminary data on $C$ albicans in a limited sample of subjects. ${ }^{13}$ The data obtained from the experimental infections of rats whose ovaries had been removed also support the idea that proteolysis may be particularly important in vaginitis. In fact, only the two proteolytic species of Candida ( $C$ albicans and $C$ parapsilosis) caused an extended and prolonged vaginal colonisation in these rats. As these two species differ in many other potential virulence factors, ${ }^{16}$ their ability to give a rat vaginal infection, comparable in extent and duration, is rather surprising, and may indicate that in experimental rat vaginitis proteinase secretion is a dominant virulence determinant. This seems at variance with human vaginitis where the concerted action of a number of virulence factors seems more likely. ${ }^{917}$

It has been established that vaginitis in rats depends on induction (by pseudoestrus) of keratinisation of vaginal epithelia, ${ }^{17}{ }^{18}$ and it is well known that the aspartyl proteinase produced by Candida sp is potently keratinolytic. ${ }^{19}$ This may also explain why none of the non-proteinase producer yeasts, including some which are clearly implicated in human vaginitis, was able to induce vaginal infection in rats. Altogether, the data of this study lead us to speculate that women harbouring acid proteinase producing species of Candida ( $C$ albicans and $C$ parapsilosis) might be at an increased risk of developing clinically active vaginitis.

This research was in part supported by the CNR (Italy) Project FATMA, Unit A Cassone. We are indebted to JD Sobel, Department of Medicine, Wayne State University, Detroit, Michigan, for a critical reading of the manuscript. AM Marella helped in the preparation of the manuscript.

1 Odds FC. Candida and candidosis. Leicester: Leicester University Press, 1988.

2 Sobel JD, Schmitt C, Meriwhether C. A new slide latex agglutination test for the diagnosis of acute Candida vaginitis. Am J Clin Pathol 1990;94:323-5.

3 Sobel JD. Epidemiology and pathogenesis of recurrent vulvovaginal candidiasis. Am J Obstet Gynecol 1985;152:924-35.

4 De Bernardis F, Lorenzini $R$, Morelli L, Cassone A. Experimental rat vaginal infection with Candida parapExperimental rat vaginal infection with Candida
silosis. FEMS Microbiol Letts 1989;65:137-42.

5 De Bernardis F, Lorenzini $R$, Verticchio $R$, Agatensi L, Cassone A. Isolation, acid proteinase secretion and
Cenardis F, Lorenzini R, Verticchio R, Agatensi L, experimental pathogenicity of Candida parapsilosis from outpatients with vaginitis. J Clin Microbiol 1989;27: $2598-603$.

6 McCormack WM, Starko KM, Zinner SH. Symptoms associated with vaginal colonization with yeast. $A m J$ Obstet Gynecol 1988;158:31-3.

7 Berg AO, Heidrich FE, Fihn SD, et al. Establishing the cause of genitourinary symptoms in women in a family practice. JAMA 1984;251:620-5.

8 Bergman JJ, Berg AO. How useful are symptoms in the diagnosis of Candida vaginitis? J Fam Pract 1983;16: diagnosis.

9 Sobel JD. Pathogenesis of Candida vulvovaginitis. In: McGinnis MR, Borgers M, eds. Current Topics in Medical Mycology. Stuttgart: Springer-Verlag, 1989:86-108.

Mycology. Stuttgart: Springer-Verlag, 1989:86-108.
10 Sweet RL. Importance of differential diagnosis in acute vaginitis. Am J Obstet Gynecol 1985;152:921-3.

11 Meyer SA, Ahearn DG, Yarrow D. GENUS 4 Candida 
Berkhout. In: Kreger NJW, ed. The yeasts, a taxonomic study. Amsterdam: Elsevier Science Publishing Inc 1984:764-7.

12 Tsuchiya T, Fukazawa $Y$, Taguchi $M$, Nakase T, Shinosa $T$. Serological aspects of yeast classification. Mycopathol Mycol Appl 1974;53:77-91.

13 Cassone A, De Bernardis F, Mondello F, Ceddia T, Agatensi L. Evidence for a correlation between proteinase secretion and vulvovaginal candidosis. J Infect Dis 1987;156: 777-83.

14 De Bernardis F, Agatensi L, Ross IK, et al. Evidence for a role for secreted aspartate proteinase of Candida albicans in vulvovaginal candidosis. J Infect Dis 1987;161:1276-83.

15 De Bernardis F, Lorenzini R, Verticchio R, Agatensi L,
Cassone A. Isolation, acid proteinase secretion, and experimental pathogenicity of Candida parapsilosis from outpatients with vaginitis. J Clin Microbiol 1989;27: 2598-603.

16 Odds FC. Candida infection: an overview. CRC Crit Rev Microbiol 1987;15:1-6.

17 Sobel JD, Muller G, Buckley $\mathrm{H}$. Critical role of germination in the pathogenesis of experimental candidal vaginitis. Infect Immun 1984;44:576-80.

$18 \mathrm{Kinsman}$ OS, Collard AE. Hormonal factors in vaginal candidiasis in rats. Infect Immun 1986;53:498-504.

19 Hattori M, Yoshiura M, Negi M, Ogawa H. Keratinolytic proteinase produced by Candida albicans. Sabouraudia proteinase produce
$1981 ; 22: 175-83$.

\section{Eponyms in pathology}

SCHWANN, Theodor (1810-1882) was a German physiologist, born near Dusseldorf. He studied medicine in Bonn, graduating in 1834. He then worked in Wurzburg and Berlin, where for five years he was a pupil of Johannes Muller (of Mullerian duct fame). It was there that he did his best work, and in 1837 published his classic declaration of cell theory in a book on the similarities of growth and structure between plants and animals. In 1839 he described the large nucleated cells of the nerve sheath of peripheral nerve fibres (Schwann cells) which are now known to produce myelin.
In the same year he was appointed Professor of physiology at Louvain, Belgium, moving to Liège in 1849 as Professor of comparative anatomy before his retirement in 1880 . Among his other achievements, he discovered pepsin (1835), showed that bile is necessary for digestion, and was the first to show that fermentation involved living organisms (being acknowledged by Pasteur for this). Soon after leaving Berlin, however, he abandoned rationalism and became a mystic, doing very little original work thereafter. 